\title{
Nomogram for predicting axillary lymph node pathological response in node-positive breast cancer patients after neoadjuvant chemotherapy
}

\section{Wenyan Wang}

Beijing Tiantan Hospital

\section{Xin Wang ( $\nabla$ xinwang@vip.126.com )}

Cancer Hospital of Chinese Academy of Medical Sciences https://orcid.org/0000-0003-1753-2786

\section{Xiang Wang}

Cancer Hospital Chinese Academy of Medical Sciences

\section{Pilin Wang}

Beijing Tiantan Hospital

\section{Research}

Keywords: Breast cancer, Neoadjuvant chemotherapy, Lymph node, Pathology response, Nomogram

Posted Date: April 8th, 2021

DOI: https://doi.org/10.21203/rs.3.rs-398841/v1

License: (9) This work is licensed under a Creative Commons Attribution 4.0 International License. Read Full License

Version of Record: A version of this preprint was published at Journal of Clinical Oncology on May 20th, 2019. See the published version at https://doi.org/10.1200/JC0.2019.37.15_suppl.e12090. 


\section{Abstract \\ Background}

Pathological complete response (pCR) of axillary lymph nodes (ALNs) is frequently achieved in patients with clinically node-positive breast cancer after neoadjuvant chemotherapy (NAC), and ALN status is an important prognostic factor for breast cancer patients. Our goal is to develop a new predictive clinical model to assess the axillary lymph node PCR rate after NAC.

\section{Methods}

A retrospective series of 547 patients who had biopsy-proven positive ALNs at diagnosis and undergoing axillary lymph node dissection from 2007 to 2014 in National Cancer Center/Cancer Hospital of Chinese Academy of Medical Sciences. We analyzed the clinicopathologic features and developed a nomogram to predict the probability of ALN pCR. Univariate assessment was performed using a logistic regression model. A multivariate logistic regression stepwise model was used to generate a nomogram to predict ALN pCR in node positive patients Variables with $\mathrm{P}<0.05$ on multivariable analysis were included in the nomogram. The adjusted area under the receiver operating characteristic curve (AUC) was calculated to quantify the ability to rank patients by risk. Internal validation was estimated using 50-50 hold out validation method. Nomogram was validated externally with the prospective cohorts of 167 patients from 2016 to 2018 of Cancer Hospital of Chinese Academy of Medical Sciences and 75 patients from 2018 to 2019 of Beijing Tiantan hospital.

\section{Results}

In retrospective study, there were $172(31.4 \%)$ patients achieved axillary pCR after NAC. Multivariate analysis indicated that clinical nodal (N) stage, estrogen receptor (ER) status and clinical response of primary tumor after NAC were significant independent predictors for axillary pCR $(P<0.05)$. The NAC nomogram was based on these three variables. In the internal validation of performance, the AUCs for the training and test sets were 0.719 and 0.753 , respectively. The nomogram was validated in external cohorts with AUCs of 0.862 and 0.766 , respectively, which demonstrated good discriminatory power in the external validation data sets.

\section{Conclusion}

We developed a nomogram to predict the likelihood of axillary $\mathrm{PCR}$ in node positive breast cancer patients after NAC. The predictive model performed well in multicenter prospective external validation. This practical tool could provide information to surgeons regarding whether to perform additional ALND after NAC. 


\section{Introduction}

Neoadjuvant chemotherapy (NAC) reduces the tumor burden in breast cancer patients and has been increasingly used in patients with axillary lymph node (ALN) metastasis [1, 2]. Nowadays, axillary lymph node dissection (ALND) has still been recommended for most patients with biopsy-proven ALD positive [3]. In patients with advanced and ALN-positive breast cancer, the pathological complete response (pCR) rate of the primary tumor is $24-46 \%$ and that of the ALNs is $30-49 \%[4,5]$. Hypothetically, ALND can be avoided in patients with axillary complete response, and the number of patients afflicted with complications such as lymphedema and arm pain can be decreased.

Sentinel lymph node biopsy (SLNB) can be used to evaluate axillary staging [6-9]. However, SLNB for patients who have received NAC is still a controversial issue. ALND is the standard axillary management for patients after NAC. The ACOSOG Z1071 study reported a false-negative rate of SLNB at $12.6 \%$ when more than two sentinel lymph nodes were examined, which exceeded the acceptable cut-off value of $10 \%$ [10].The SNFNAC study reported an identification rate of $87.6 \%$ and an false negative rate of $8.4 \%$ in patients with node-positive breast cancer after NAC [7]. Accurate prediction of achieving axillary response after NAC is important in establishing a treatment plan for patients with node positive breast cancer. Therefore, in the present study, we sought to identify possible predictors and construct a nomogram for predicting pCR of ALN after NAC among biopsy proven node positive breast cancer patients, which will increase the accuracy of SLNB after NAC. Combining SLNB and nomogram prediction, patients with high likelihood of lymph nodes PCR can avoid ALN dissection.

\section{Methods}

We performed a retrospective review of 547 patients who had biopsy-proven positive ALNs at diagnosis and undergoing axillary lymph node dissection from January 1, 2007 to September 30, 2014 in National Cancer Center/Cancer Hospital of Chinese Academy of Medical Sciences (CHCAMS). Our study population involved the following criteria: (i) histologically confirmed primary invasive breast carcinoma; (ii) ALNs metastases diagnosed by fine needle aspiration (FNA); (iii) treated with NAC before surgery; (iv) underwent ALN dissection after NAC. Exclusion criteria included: (i) patients with distant metastases; (ii) patients with negative ALNs before NAC. The retrospective series was used to develop a predictive nomogram. The nomogram was then validated externally with two prospective cohorts, 167 patients from May 1, 2016 to January 31, 2018 in National Cancer Center/Cancer Hospital of Chinese Academy of Medical Sciences, and 75 patients in Beijing Tiantan Hospital affliated to Capital Medical University (BTH) from Jan 1st, 2018 to April 30th, 2019. The prospective trial (http://www.chictr.org/cn/, identifier ChiCTR1800014968) was approved by the institutional review board (IRB) of Cancer Hospital, Chinese Academy of Medical Sciences and IRB of Beijing Tiantan Hospital affliated to Capital Medical University. The informed consent was waived in the retrospective study. Patients accrued to the prospective cohort were required to sign the study consent before the surgery date. 
Clinical staging, ultrasonography and magnetic resonance imaging before and after NAC were performed. TNM classification was based on the AJCC Cancer Staging Manual, Eighth Edition. We also evaluated the MRI and US after NAC to assess the clinical response of primary tumors and ALNs. All patients received NAC and standard chemotherapy regimens containing anthracyclines and taxanes that were given according to the guidelines or within ongoing protocols. Trastuzumab was added to taxane-based chemotherapy for patients with human epidermal receptor 2 (HER2)-overexpressing cancer. Altered or interrupted treatment was recorded with the reason for disruption. All patients underwent either breastconserving surgery (BCS) or mastectomy followed by a standard ALND of levels I and II.

Clinical response was assessed according to response evaluation criteria in solid tumors guidelines [11]. Complete response (CR) was defined as the absence of evidence of a palpable tumor in breast and/or no visible tumor on MRI or US after NAC. Partial response (PR) stands for at least $30 \%$ decrease in lesion(s). Progressive disease (PD) stands for $20 \%$ increase of lesions. Stable disease (SD) indicated that neither PR nor PD criteria were met. Pathologic response was assessed after completion of NAC using MillerPayne Grading system [12]. Surgical specimens with no histological evidence of invasive carcinoma in the breast and of metastatic carcinoma cells in removed lymph nodes were classified as PCR.

The original blocks were stained for estrogen receptor (ER), progesterone receptor (PR), and human epidermal growth factor receptor 2 (HER2) antigen. Immunohistochemical staining positivity for ER and PR was defined as $1 \%$ or more nuclear staining. The HER2 assessment was performed as per the guidelines of the American Society of Clinical Oncology and the College of American Pathologists. Samples were considered positive if they scored $3+$ and negative if they scored $1+$. For patients with a score of $2+$, the samples were further assayed using fluorescence in situ hybridization and considered positive if the ratio of HER2 signal to centromeric probe for chromosome 17 (CEP17) was greater than 2.2 [13].

Descriptive analysis was performed for clinicopathological features of patients. Univariate assessment was performed using a logistic regression model. A multivariate logistic regression stepwise model was used to generate a nomogram to predict ALN pCR in node positive patients Variables with $\mathrm{P}<0.05$ on multivariable analysis were included in the nomogram. The adjusted area under the receiver operating characteristic curve (AUC) was calculated to quantify the ability to rank patients by risk. Internal validation was estimated using $50-50$ hold out validation method. Nomogram was validated externally with the prospective cohort of 167 patients. All teats were two-sided, and $\mathrm{P} \leq 0.05$ indicated statistical significance. Statistical analysis was performed using SPSS, version 22.0 (SPSS Inc., Chicago, IL, U.S.) and $\mathrm{R}$ version 3.5.3 (R Foundation, Vienna, Austria). The related $\mathrm{R}$ packages were applied in construction and assessment of the nomogram, including 'rms', 'glmnet', 'Hmisc', 'generalhoslem', 'ggplot2' and'Dca.R'.

\section{Results}

\section{Patient characteristics}


The baseline characteristics showed 547 patients had positive ALNs and underwent ALND (Table 1). The median age was 52 years (range $24-75$ years). 172 (31.4\%) patients achieved axillary lymph node pCR after NAC. 
Table 1

Demographic and clinicopathologic characteristics of patients in the retrospective series

\begin{tabular}{|c|c|c|c|}
\hline Variable & LNs non-pCR $n=375(\%)$ & $\begin{array}{l}\text { LNs pCR } \\
n=172(\%)\end{array}$ & P value \\
\hline Age & & & 0.092 \\
\hline$\leq 50$ & $150(40.0)$ & $82(47.7)$ & \\
\hline$>50$ & $225(60.0)$ & $90(52.3)$ & \\
\hline Pathological type & & & 0.969 \\
\hline IDC & $360(96.0)$ & 165 (95.9) & \\
\hline Others ${ }^{*}$ & $15(4.0)$ & $7(4.1)$ & \\
\hline Clinical T stage & & & 0.728 \\
\hline T1 & $8(2.1)$ & $6(3.5)$ & \\
\hline T2 & $165(44.0)$ & $72(41.9)$ & \\
\hline T3 & $122(32.5)$ & $60(34.9)$ & \\
\hline T4 & $80(21.3)$ & 34 (19.7) & \\
\hline Clinical N stage & & & 0.013 \\
\hline $\mathrm{N} 1$ & $291(77.6)$ & 149 (86.6) & \\
\hline N2 & $84(22.1)$ & $23(13.4)$ & \\
\hline Primary tumor response & & & $<0.001$ \\
\hline $\mathrm{CR}$ & $60(16.0)$ & $83(48.3)$ & \\
\hline PR and SD & $315(84.0)$ & $89(51.7)$ & \\
\hline Histological grade & & & 0.865 \\
\hline I & $13(3.5)$ & $6(3.5)$ & \\
\hline ॥ & $257(68.5)$ & $104(60.5)$ & \\
\hline III & $105(28.0)$ & $62(36.0)$ & \\
\hline ER & & & 0.002 \\
\hline Negative & $119(31.7)$ & $58(33.7)$ & \\
\hline Positive & $233(62.1)$ & $59(34.3)$ & \\
\hline
\end{tabular}

*Others, invasive lobular carcinoma, papillary carcinoma, adenoid cystic carcinoma, mucinous carcinoma; 


\begin{tabular}{|llll|}
\hline Variable & LNs non-pCR $n=375(\%)$ & $\begin{array}{l}\text { LNs pCR } \\
\mathbf{n}=\mathbf{1 7 2}(\%)\end{array}$ & P value \\
\hline Unknown & $23(6.2)$ & $55(32.0)$ & \\
\hline PR & & & \\
\hline Negative & $131(34.9)$ & $56(32.5)$ & \\
\hline Positive & $221(58.9)$ & $61(35.5)$ & \\
\hline Unknown & $23(6.2)$ & $55(32.0)$ & \\
\hline HER2 & & $92(53.5)$ & \\
\hline Negative & $270(72.0)$ & $25(14.5)$ & \\
\hline Positive & $80(21.3)$ & $55(32.0)$ \\
\hline Unknown & $25(6.7)$ & & \\
\hline $\begin{array}{l}* \text { Others, invasive lobular carcinoma, papillary carcinoma, adenoid cystic carcinoma, mucinous } \\
\text { carcinoma; }\end{array}$ & \\
\hline
\end{tabular}

On univariate analysis, clinical T stage, $\mathrm{N}$ stage, primary tumor response and estrogen receptor status were significantly associated with the likelihood of axillary lymph node $\mathrm{PCR}(\mathrm{P}<0.05$, Table 2$)$. On multivariate stepwise logistic regression analysis, clinical $N$ stage $(P=0.002$; odds ratio [OR], $2.408 ; 95 \%$ confidence interval [Cl], 1.383-4.194), primary tumor response $(P<0.001 ; O R, 0.189 ; 95 \% \mathrm{Cl}, 0.123-$ 0.292), and estrogen receptor status $(P=0.025 ; O R, 0.530,95 \% \mathrm{Cl}, 0.304-0.925)$ were independent predictors of ALN pCR (Table 3). 
Table 2

Univariates analysis of axillary lymph node $\mathrm{PCR}$ in the retrospective patient series

\begin{tabular}{|c|c|c|c|}
\hline Variable & OR & $95 \% \mathrm{Cl}$ & $P$ value \\
\hline \multicolumn{4}{|l|}{ Age } \\
\hline$\leq 50$ & 1 & & \\
\hline$>50$ & 0.592 & $0.442 \sim 0.998$ & 0.442 \\
\hline \multicolumn{4}{|c|}{ Pathological type } \\
\hline IDC & 1 & & \\
\hline Others ${ }^{*}$ & 1.184 & $0.105 \sim 13.33$ & 0.891 \\
\hline \multicolumn{4}{|c|}{ Clinical T stage } \\
\hline T1 & 1 & & \\
\hline T2 & 1.839 & $1.414 \llbracket 2.392$ & $<0.001$ \\
\hline T3 & 3.437 & $2.674 \llbracket 4.417$ & $<0.001$ \\
\hline T4 & 9.221 & $9.197 \rrbracket 11.81$ & $<0.001$ \\
\hline \multicolumn{4}{|c|}{ Clinical N stage } \\
\hline N1 & 1 & & \\
\hline N2 & 3.462 & $1.792 \sim 6.668$ & $<0.001$ \\
\hline \multicolumn{4}{|c|}{ Primary tumor response } \\
\hline $\mathrm{CR}$ & 1 & & \\
\hline PR and SD & 1.082 & $1.011 \otimes 1.158$ & 0.023 \\
\hline \multicolumn{4}{|c|}{ Histological grade } \\
\hline I & 1 & & 0.801 \\
\hline II & 1.216 & $0.704 \sim 1.747$ & \\
\hline III & 1.585 & $0.52 \rrbracket 2.03$ & \\
\hline \multicolumn{4}{|l|}{ ER } \\
\hline Negative & 1 & & \\
\hline Positive & 0.554 & $0.287 \sim 0.807$ & $<0.001$ \\
\hline PR & & & \\
\hline
\end{tabular}




\begin{tabular}{|llll|}
\hline Variable & OR & $95 \% \mathrm{Cl}$ & P value \\
\hline Negative & 1 & & \\
\hline Positive & 0.568 & $0.296 \sim 0.890$ & 0.039 \\
\hline Unknown & 0.844 & $0.516 \sim 1.090$ & 0.089 \\
\hline HER2 & & & \\
Negative & 1 & & 0.504 \\
\hline Positive & 1.215 & $0.649 \sim 2.410$ & 0.81 \\
\hline Unknown & 0.347 & $0.125 \sim 1.442$ & \\
\hline $\begin{array}{l}\text { Note: IDC, invasive ductal carcinoma; Clinical T stage, clinical tumor stage; Clinical N stage, clinical } \\
\text { nodal stage; CR, complete response; PR, partial response; SD, stable disease; ER, estrogen receptor; } \\
\text { PR, progesterone receptor; HER2, human epithelia growth factor receptor 2. }\end{array}$ & \\
\hline
\end{tabular}

Table 3

Logistic regression analysis using backward stepwise likelihood ratio method in the retrospective patient series

\begin{tabular}{|llllll|}
\hline Variable & Coefficient & SE & Wald & OR (95\%Cl) & P value \\
\hline Age & -0.391 & 0.205 & 3.647 & $0.677(0.453-1.010)$ & 0.056 \\
\hline Clinical T stage & & & & & 0.420 \\
\hline T1 & 0.533 & 0.658 & 0.655 & $1.704(0.469-6.189)$ & \\
\hline T2 & -0.260 & 0.280 & 0.858 & $0.771(0.445-1.336)$ & \\
\hline T3 & 0.033 & 0.288 & 0.013 & $1.033(0.587-1.819)$ & \\
\hline Clinical N stage & 0.879 & 0.283 & 9.639 & $2.408(1.383-4.194)$ & 0.002 \\
\hline Tumor type & -0.292 & 0.515 & 0.322 & $0.747(0.272-2.049)$ & 0.570 \\
\hline Histologic grade & -0.180 & 0.629 & 0.082 & $0.835(0.444-1.174)$ & 0.775 \\
\hline Primary tumor response & -1.762 & 0.231 & 58.15 & $0.189(0.123-0.292)$ & $<0.001$ \\
\hline ER & -0.635 & 0.284 & 4.991 & $0.530(0.304-0.925)$ & 0.025 \\
\hline PR & -0.031 & 0.284 & 0.012 & $0.970(0.555-1.694)$ & 0.915 \\
\hline HER2 & 0.331 & 0.231 & 2.047 & $1.392(0.885-2.189)$ & 0.153 \\
\hline $\begin{array}{l}\text { Note: SE, standard error; Cl, confidence interval; Clinical T stage, clinical tumor stage; Clinical N stage, } \\
\text { clinical nodal stage; ER, estrogen receptor; PR, progesterone receptor; HER2, human epithelia growth } \\
\text { factor receptor 2. }\end{array}$ & & & & & \\
\hline
\end{tabular}


Table 4

Patients characteristics in External Validation Group

\begin{tabular}{|c|c|c|c|c|}
\hline Variable & $\begin{array}{l}\text { Patients of CHCAMS } \\
n=167(\%)\end{array}$ & $\begin{array}{l}\text { Patients of BTH } \\
n=74(\%)\end{array}$ & Chi-square & $P$ value \\
\hline Age & $45.7 \pm 4.76$ & $53.7 \pm 3.92$ & & \\
\hline Tumor stage & & & 15.46 & 0.002 \\
\hline cT1 & $7(4.2)$ & $8(10.8)$ & & \\
\hline cT2 & $69(41.3)$ & $45(60.8)$ & & \\
\hline cT3 & $74(44.3)$ & $16(21.6)$ & & \\
\hline cT4 & $17(10.2)$ & $5(6.8)$ & & \\
\hline $\mathrm{N}$ stage & & & 0.11 & 0.916 \\
\hline $\mathrm{cN} 1$ & $105(62.9)$ & $46(62.2)$ & & \\
\hline $\mathrm{cN} 2$ & $62(37.1)$ & $28(37.8)$ & & \\
\hline Tumor Grade & & & 2.05 & 0.359 \\
\hline I & $9(5.7)$ & $2(2.7)$ & & \\
\hline II & $98(58.8)$ & $50(67.6)$ & & \\
\hline III & $60(35.5)$ & $22(29.7)$ & & \\
\hline $\mathrm{HR}$ & & & 0.284 & 0.594 \\
\hline Negative & $63(37.7)$ & 31 (41.3) & & \\
\hline Positive & $104(62.3)$ & $44(58.7)$ & & \\
\hline HER2 & & & 9.000 & 0.003 \\
\hline Negative & $100(59.9)$ & $59(79.7)$ & & \\
\hline Positive & $67(40.1)$ & 15 (20.3) & & \\
\hline
\end{tabular}

\section{Nomogram}

A nomogram was developed using 4 variables: clinical T stage, clinical nodal stage, primary tumor response and HR (Figure 1). The total sum for each variable was located on a "total points" line, and a line was drawn downward to calculate the probability of axillary pCR. In the internal validation of performance, the AUCs for the training and test sets were $0.719(95 \% \mathrm{Cl} 0.638-0.771)$ and $0.753(95 \% \mathrm{Cl}$ 
0.704-0.791), respectively, demonstrating that the nomogram provides fairly precise predictions of ALN pathological response after NAC (Figure 2).

\section{External Validation}

The prospective series included 242 patients with positive LNs before NAC were obtained for external validation of the nomogram, 167 patients from Cancer Hospital, Chinese Academy of Medical Sciences (CHCAMS) and 75 patients from Beijing Tiantan Hospital (BTH). The median age was $45.7 \pm 4.76$ years and $53.7 \pm 3.92$ years, respectively. In CHCAMS series, 71 patients $(42.5 \%)$ had breast tumor complete response and 62 patients (37.1\%) had ALNs pathology complete response. While in BTH validation group, 19 patients $(25.3 \%)$ had breast complete response and 42 patients $(56.0 \%)$ had ALNs pathology complete response (Table 3). When the nomogram applied to the prospective series, the AUCs were $0.862(95 \% \mathrm{Cl}$ 0.793-0.911) and 0.766 (95\% Cl 0.616-0.826), respectively (Figure 3), which demonstrated good discriminatory power in the external validation data sets.

\section{Discussion}

Surgical management of axillary after NAC is closely related to pathological response. With the development of chemotherapy regiment and targeted anti-HER2 treatment, the primary tumor and axillary pCR rates have increased substantially. Since the application of SLNB after NAC for assessing axillary status, the standard treatment ALND may be omitted in axillary pCR patients after accurately identified. Built on the present researches, we constructed a nomogram to predict ALN pCR in node positive patients after NAC. To avoid bias, we performed it in separate comprehensive institutions. In this study we performed a registered prospectively database to demonstrate a nomogram to predict axillary pCR after NAC and we performed two prospective series from different centers to validate the probability of nomogram.

In this study we performed a registered prospectively database to demonstrate a nomogram to predict axillary pCR after NAC. Two prospective series from different centers were then carried out to validate the accuracy of the nomogram. We analyzed 547 patients with biopsy-proven ALN positive breast cancer. Among them, 172 (31.4\%) patients achieved axillary pCR, which is slightly higher than the results reported in the studies of Gonzalez-Angulo et al [14] and Kida K et al [15]. Based on the multivariate analysis, we identified that clinical nodal $(\mathrm{N})$ stage, estrogen receptor (ER) status and clinical response of the primary tumor after NAC were significant independent predictors for axillary $P C R(P<0.05)$. Histologic grade and HER2 status did not show statistically significant in the multivariate logistic regression analysis. In addition, biological subtype was also associated with PCR. Based on the data from Z1071 trial, Boughey et al found that the pCR rate was $21.1 \%$ in HR+/HER2- patients, but in patients with HR-/HER2-, it was 49.4\% $(P<0.0001)$ [16]. In our study, ER negative patients were the most likely to achieve pCR, which was consistent with the previous study.

The nomogram is used as prediction tool to provide individualized estimates of risk $[17,18]$. It is concise and powerful for predicting ALN pCR, which could help to assess the actual ALN status and increase the 
accuracy of SLNB. Built on the present researches, we constructed a nomogram to predict ALN pCR in node positive patients after NAC. To avoid bias, we performed it in separate comprehensive institutions. Some researchers also have evaluated nomograms for predicting axillary status in patients with breast cancer $[9,19]$. The result of SENTINA showed that in patients whose axillary have downstage to cNO after NAC, the identification rate (IR) was $80.1 \%$ and false negative rate (FNR) was $14.2 \%$ [20]. The ACOSOG Z1071 study reported a FNR of SLNB at $12.6 \%$ when more than two SLNs were examined, which exceeded the acceptable cut-off value of $10 \%[21,22]$. Based on our nomogram, patients with high points were more likely to show ALN pCR. Combination of imaging tests, patients could safely avoid receiving ALN dissection [23]. The evaluation of axillary offering prognostic information about breast cancer. Recent studies have showed no residual invasive cancer in breast and axillary was used to provide a better outcome [24-26]. Among patients with cytologically proven axillary lymph node (ALN) metastases, survival was improved in which achieving ALN pCR [26]. While the nomogram may also be helpful in the communications between patients and oncologists.

Our study has some limitations. The retrospective data may have selection bias since it included patients who have been monitored up until 2007. Additionally, chemotherapy might be a confounding factor, for patients from different hospitals did is not given homogeneous NCT. In addition, the National Comprehensive Cancer Network (NCCN) guidelines have incorporated a comment that marking biopsied lymph nodes to document their removal can decrease the FNR of SLN after NAC. However, clipping of ALNs at initial percutaneous biopsy is still a challenge and this procedure has not been established in China. Our study was conducted only in the Chinese population and the result still needs to be validated in larger population in different regions.

In conclusion, we constructed a high discrimination and calibration ability nomogram for predicting postNAC axillary lymph node $\mathrm{pCR}$, which included five comprehensive predictors. With the nomogram, we can predict post-NAC lymph node status accurately and conduct precise surgery for patients with a high probability of achieving axillary $\mathrm{pCR}$.

\section{Abbreviations}

PCR

Pathological complete response

ALN

axillary lymph nodes

ALND

axillary lymph node dissection

NAC

neoadjuvant chemotherapy

AUC

Adjusted area under the characteristic curve

SLNB 
Sentinel lymph node biopsy

OR

Odds ratio

$\mathrm{Cl}$

Confidence interval

ER

Estrogen receptor

PR

Progesterone receptor

HER2

Human epidermal growth factor receptor 2

\section{Declarations}

\section{Ethics approval and consent to participate}

The study was approved by the institutional review board (IRB) of Cancer Hospital, Chinese Academy of Medical Sciences and IRB of Beijing Tiantan Hospital affliated to Capital Medical University.

\section{Consent for publication}

Not applicable.

\section{Availability of data and materials}

The datasets used and analyzed during the current study are available from the corresponding author on reasonable request.

\section{Competing interests}

The authors declare that they have no competing interests.

\section{Funding}

This research was supported by Grants from the Capital Health Specialized Development Research, Beijing Municipal Health Bureau (Grants No. 2016-4-4026), CAMS Innovation Fund for Medical Sciences (CIFMS) (Grant No.2016-I2M-1-001 and Grant No.2017-I2M-3-004), National Natural Science Foundation of China (Grant No. 81602343) and Youth Research Fund of Beijing Tiantan Hospital (Grant No. 2017YQN-09)

\section{Authors' Contributions}

WYW offered the idea of this study, analyzed the patient data, and drafted the manuscript. XW and WYW performed the statistical analysis and revised the manuscript. XW revised the manuscript and did a lot of 
work in data collection. XW and PLW was the supervisor of this study and was a major contributor in writing the manuscript. All authors read and approved the final manuscript.

\section{Acknowledgement}

Not applicable

\section{Author information}

Affiliations

${ }^{11}$ Department of Breast surgery, Beijing Tiantan Hospital, Capital Medical University, Beijing, 100070, China

Wenyan Wang \& Pilin Wang

${ }^{2}$ Department of Breast Surgical Oncology, National Cancer Center/Cancer Hospital of Chinese Academy of Medical Sciences, Beijing, 100021, China

Xin Wang \& Xiang Wang

Corresponding author

Correspondence to Xin Wang and Pilin Wang

\section{References}

[1] Fisher B, Bryant J, Wolmark N, et al. Effect of preoperative chemotherapy on the outcome of women with operable breast cancer. J Clin Oncol. 1998. 16(8): 2672-85.

[2] Fisher ER, Wang J, Bryant J, Fisher B, Mamounas E, Wolmark N. Pathobiology of preoperative chemotherapy: findings from the National Surgical Adjuvant Breast and Bowel (NSABP) protocol B-18. Cancer. 2002. 95(4): 681-95.

[3] Axelsson CK, Mouridsen HT, Zedeler K. Axillary dissection of level I and II lymph nodes is important in breast cancer classification. The Danish Breast Cancer Cooperative Group (DBCG). Eur J Cancer. 1992. 28A(8-9): 1415-8.

[4] Cortazar P, Zhang L, Untch M, et al. Pathological complete response and long-term clinical benefit in breast cancer: the CTNeoBC pooled analysis. Lancet. 2014. 384(9938): 164-72.

[5] Kim WH, Kim HJ, Park HY, et al. Axillary Pathologic Complete Response to Neoadjuvant Chemotherapy in Clinically Node-Positive Breast Cancer Patients: A Predictive Model Integrating the Imaging Characteristics of Ultrasound Restaging with Known Clinicopathologic Characteristics. Ultrasound Med Biol. 2019. 45(3): 702-709. 
[6] Mansel RE, Fallowfield L, Kissin M, et al. Randomized multicenter trial of sentinel node biopsy versus standard axillary treatment in operable breast cancer: the ALMANAC Trial. J Natl Cancer Inst. 2006. 98(9): 599-609.

[7] Boileau JF, Poirier B, Basik M, et al. Sentinel node biopsy after neoadjuvant chemotherapy in biopsyproven node-positive breast cancer: the SN FNAC study. J Clin Oncol. 2015. 33(3): 258-64.

[8] Caudle AS, Yang WT, Krishnamurthy S, et al. Improved Axillary Evaluation Following Neoadjuvant Therapy for Patients With Node-Positive Breast Cancer Using Selective Evaluation of Clipped Nodes: Implementation of Targeted Axillary Dissection. J Clin Oncol. 2016. 34(10): 1072-8.

[9] Kantor O, Sipsy LM, Yao K, James TA. A Predictive Model for Axillary Node Pathologic Complete Response after Neoadjuvant Chemotherapy for Breast Cancer. Ann Surg Oncol. 2018. 25(5): 1304-1311.

[10] Boughey JC, Suman VJ, Mittendorf EA, et al. Factors affecting sentinel lymph node identification rate after neoadjuvant chemotherapy for breast cancer patients enrolled in ACOSOG Z1071 (Alliance). Ann Surg. 2015. 261(3): 547-52.

[11] Eisenhauer EA, Therasse P, Bogaerts $J$, et al. New response evaluation criteria in solid tumours: revised RECIST guideline (version 1.1). Eur J Cancer. 2009. 45(2): 228-47.

[12] Ogston KN, Miller ID, Payne S, et al. A new histological grading system to assess response of breast cancers to primary chemotherapy: prognostic significance and survival. Breast. 2003. 12(5): 320-7.

[13] Edge SB, Compton CC. The American Joint Committee on Cancer: the 7th edition of the AJCC cancer staging manual and the future of TNM. Ann Surg Oncol. 2010. 17(6): 1471-4.

[14] Tausch C, Konstantiniuk P, Kugler F, et al. Sentinel lymph node biopsy after preoperative chemotherapy for breast cancer: findings from the Austrian Sentinel Node Study Group. Ann Surg Oncol. 2008. 15(12): 3378-83.

[15] Kida K, Ishikawa T, Yamada A, et al. A prospective feasibility study of sentinel node biopsy by modified Indigocarmine blue dye methods after neoadjuvant chemotherapy for breast cancer. Eur J Surg Oncol. 2015. 41(4): 566-70.

[16] Boughey JC, McCall LM, Ballman KV, et al. Tumor biology correlates with rates of breast-conserving surgery and pathologic complete response after neoadjuvant chemotherapy for breast cancer: findings from the ACOSOG Z1071 (Alliance) Prospective Multicenter Clinical Trial. Ann Surg. 2014. 260(4): 608-14; discussion 614-6.

[17] Kerr KF, Brown MD, Zhu K, Janes H. Assessing the Clinical Impact of Risk Prediction Models With Decision Curves: Guidance for Correct Interpretation and Appropriate Use. J Clin Oncol. 2016. 34(21): 2534-40. 
[18] Balachandran VP, Gonen M, Smith JJ, DeMatteo RP. Nomograms in oncology: more than meets the eye. Lancet Oncol. 2015. 16(4): e173-80.

[19] Choi HJ, Ryu JM, Kim I, et al. Nomogram for accurate prediction of breast and axillary pathologic response after neoadjuvant chemotherapy in node positive patients with breast cancer. Ann Surg Treat Res. 2019. 96(4): 169-176.

[20] Kuehn T, Bauerfeind I, Fehm T, et al. Sentinel-lymph-node biopsy in patients with breast cancer before and after neoadjuvant chemotherapy (SENTINA): a prospective, multicentre cohort study. Lancet Oncol. 2013. 14(7): 609-18.

[21] Boughey JC, Suman VJ, Mittendorf EA, et al. Sentinel lymph node surgery after neoadjuvant chemotherapy in patients with node-positive breast cancer: the ACOSOG Z1071 (Alliance) clinical trial. JAMA. 2013. 310(14): 1455-61.

[22] Boughey JC, Ballman KV, Le-Petross HT, et al. Identification and Resection of Clipped Node Decreases the False-negative Rate of Sentinel Lymph Node Surgery in Patients Presenting With Node-positive Breast Cancer (T0-T4, N1-N2) Who Receive Neoadjuvant Chemotherapy: Results From ACOSOG Z1071 (Alliance). Ann Surg. 2016. 263(4): 802-7.

[23] Santamaría G, Bargalló X, Ganau S, et al. Multiparametric MR imaging to assess response following neoadjuvant systemic treatment in various breast cancer subtypes: Comparison between different definitions of pathologic complete response. Eur J Radiol. 2019. 117: 132-139.

[24] Broglio KR, Quintana M, Foster M, et al. Association of Pathologic Complete Response to Neoadjuvant Therapy in HER2-Positive Breast Cancer With Long-Term Outcomes: A Meta-Analysis. JAMA Oncol. 2016. 2(6): 751-60.

[25] Rouzier R, Extra JM, Klijanienko J, et al. Incidence and prognostic significance of complete axillary downstaging after primary chemotherapy in breast cancer patients with T1 to T3 tumors and cytologically proven axillary metastatic lymph nodes. J Clin Oncol. 2002. 20(5): 1304-10.

[26] Hennessy BT, Hortobagyi GN, Rouzier R, et al. Outcome after pathologic complete eradication of cytologically proven breast cancer axillary node metastases following primary chemotherapy. $\mathrm{J}$ Clin Oncol. 2005. 23(36): 9304-11.

\section{Figures}


Points

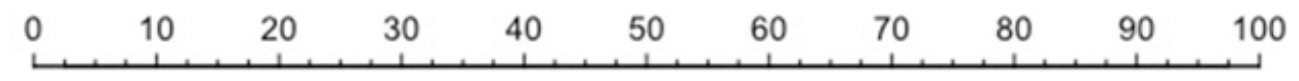

age

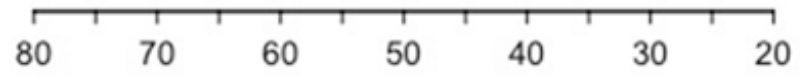

ER

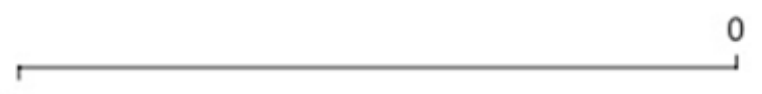

1

PR

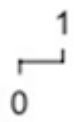

HER2

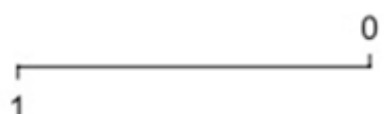

T_stage

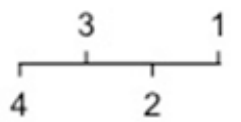

N_stage

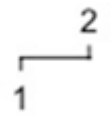

breast_response

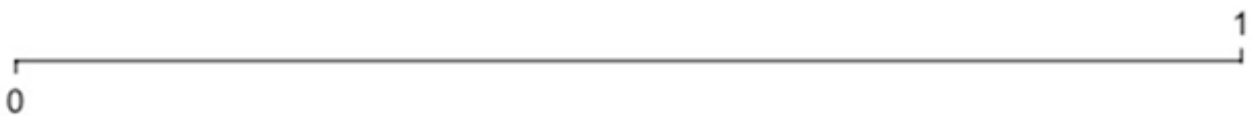

Total Points

\begin{tabular}{lllllllllllllll}
\hline 0 & 20 & 40 & 60 & 80 & 100 & 120 & 140 & 160 & 180 & 200 & 220 & 240 & 260 & 280
\end{tabular}

probability of axillary pCR

0.1

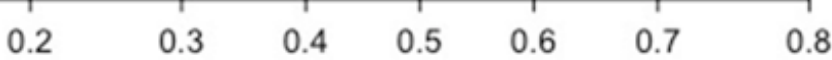

\section{Figure 1}

Nomogram to predict Axillary lymph node pathological complete response in patients with positive lymph node before NAC. 


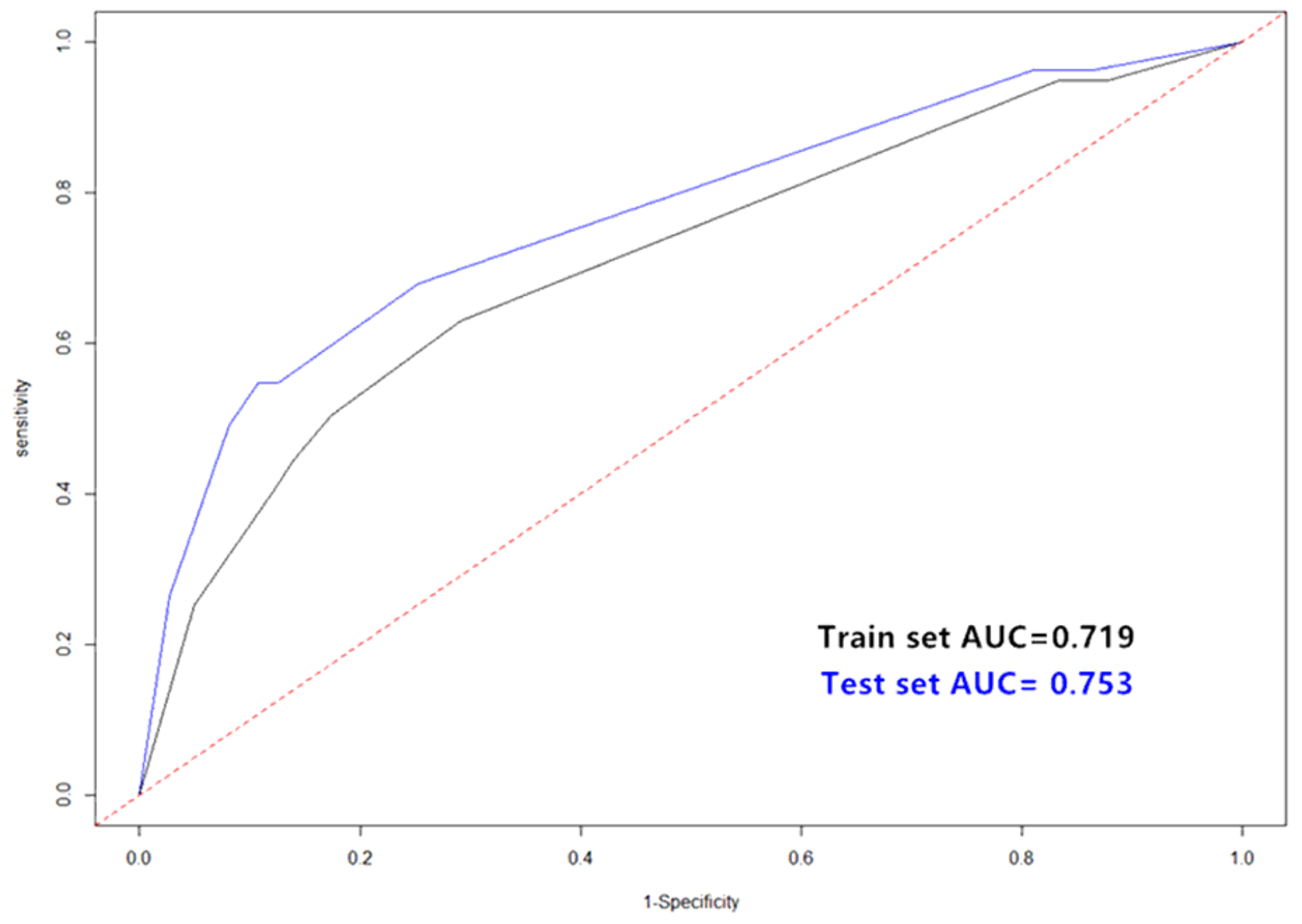

Figure 2

Receiver Operating Characteristics (ROC) Curve for Nomogram in the training and testing sets. The area under the curve (AUC) were 0.719 and 0.753 respectively. 

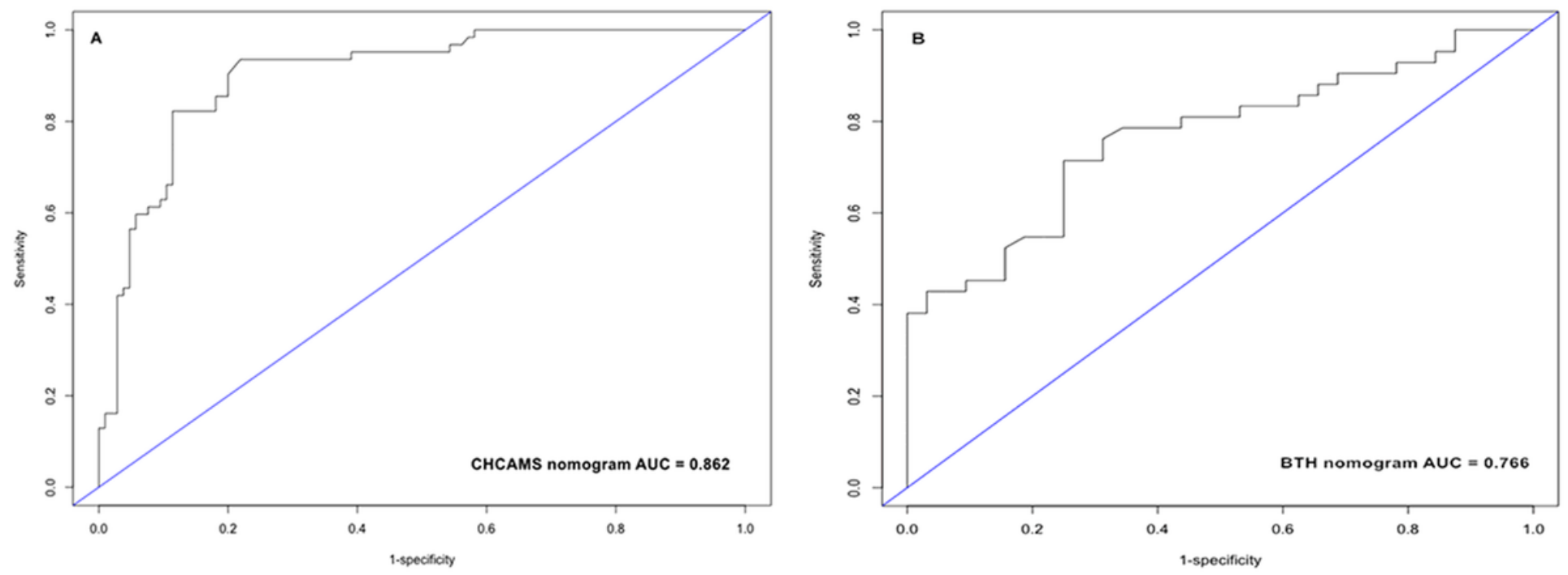

Figure 3

Receiver Operation characteristics (ROC) Curves for discrimination of External Validation sets. The areas under the curve (AUC) were 0.862 (95\% Cl 0.793-0.911) (Fig.3A) and 0.766 (95\% Cl 0.616-0.826) (Fig 3B) respectively. 